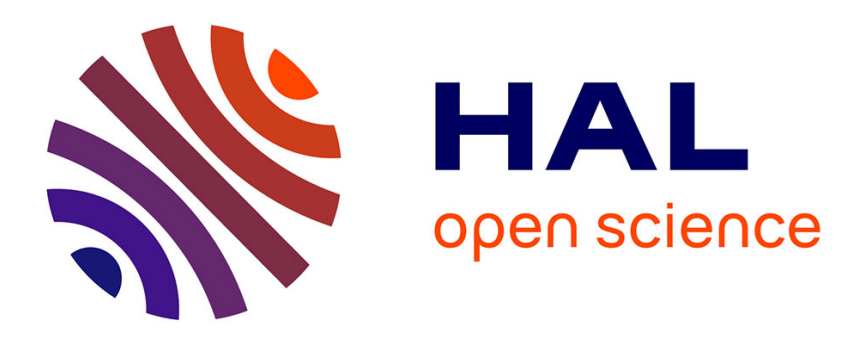

\title{
Le concept d'attachement au lieu : état de l'art et voies de recherche dans le contexte du lieu de loisirs.
}

\author{
Alain Debenedetti
}

\section{To cite this version:}

Alain Debenedetti. Le concept d'attachement au lieu: état de l'art et voies de recherche dans le contexte du lieu de loisirs.. Revue management \& avenir, 2005, 5 (juin), pp.151-160. hal-01214660

\author{
HAL Id: hal-01214660 \\ https://hal.science/hal-01214660
}

Submitted on 12 Oct 2015

HAL is a multi-disciplinary open access archive for the deposit and dissemination of scientific research documents, whether they are published or not. The documents may come from teaching and research institutions in France or abroad, or from public or private research centers.
L'archive ouverte pluridisciplinaire HAL, est destinée au dépôt et à la diffusion de documents scientifiques de niveau recherche, publiés ou non, émanant des établissements d'enseignement et de recherche français ou étrangers, des laboratoires publics ou privés. 
Le concept d'attachement au lieu : état de l'art et voies de recherche dans le contexte du lieu de loisirs.

\section{Alain DEBENEDETTI}

Alain Debenedetti est doctorant à l'ESSEC et rattaché à l'Université de ParisDauphine, centre DMSP.

Programme Doctoral ESSEC

Avenue Bernard Hirsch

BP 105

95021 Cergy-Pontoise cedex

Université de Paris-Dauphine

Centre de recherche DMSP

Place du Maréchal de Lattre de Tassigny

75775 Paris cedex 16

contact : alain.debenedetti@dauphine.fr 
Le concept d'attachement au lieu : état de l'art et voies de recherches dans le contexte du lieu de loisirs.

\title{
Résumé
}

L'objectif de cet article est de montrer la pertinence du concept d'attachement au lieu pour qualifier la relation affective entre un consommateur et un lieu de service. Cet article résume l'ensemble des résultats obtenus par les chercheurs et souligne les différentes voies de recherches qu'il reste à explorer, en particulier dans le contexte des lieux de loisirs.

Place attachment concept : review of the literature and perspectives in the leisure places context

\begin{abstract}
The aim of this article is to enlight the relevancy of the concept of place attachment in order to qualify the affective relationship between consumers and service areas. This paper reviews the overall results obtained so far by researchers and highlights the different research avenues left to explore, particularly in the framework of leisure places.
\end{abstract}




\section{Le concept d'attachement au lieu : état de l'art et voies de recherche dans le contexte du lieu de loisirs.}

Longtemps resté dans l'ombre d'une recherche focalisée sur l'adaptation aux nouvelles situations dans un monde fondé sur l'instabilité et le changement, l'attachement au lieu a récemment pris de l'ampleur avec l'étude des relations entre l'individu et son lieu de naissance (Low et Altman, 1992). Il est envisagé dans de nombreuses disciplines comme les sciences des loisirs ou la psychologie environnementale et à travers de nombreuses approches. Dans le cadre d'une relation entre un consommateur et un lieu de service, ce concept n'a, à notre connaissance, jamais fait l'objet de recherches, même si Belk (1992) soulignait déjà l'intérêt de le mobiliser il y a plus de 10 ans. Par ailleurs, la diversité des approches et l'apparente faiblesse des échanges entre disciplines autour du construit n'ont pas favorisé la production de connaissances nouvelles. Elles font de l'attachement au lieu un construit dont les mécanismes restent à explorer, même si la littérature s'accorde sur ses caractéristiques (Schultz-Kleine et Menzel Baker, 2004) et permet d'en donner une définition : il s'agit d'un lien affectif positif et identitaire entre un consommateur et un lieu spécifique (le lieu devient une extension du soi) qui a pour nature de durer même s’il est susceptible de varier en intensité.

Aujourd'hui, face à une concurrence qui limite l'impact des variables commerciales utilitaires, la prise en compte des facteurs affectifs dans les lieux de service devient un enjeu majeur même si la relation qui s'établit entre un consommateur et un lieu de service, complexe, fait intervenir un ensemble d'affects et de cognitions (Filser, 1996). De plus, l'existence d'un lien affectif, fortement ancré dans le moi du consommateur, qui fait du consommateur un ambassadeur du lieu, implique une résistance au changement et rend la séparation avec le lieu difficile, permet d'envisager l'attachement a priori comme une forme de comportement de fidélité très spécifique, intéressant donc les managers pour des raisons évidentes. Enfin, si de nombreux travaux académiques s'intéressent à l'aspect affectif de l'expérience de shopping ou au rôle de l'affect dans la consommation, peu de recherches s'intéressent aux différents types d'affects qui peuvent s'établir entre un individu et un lieu.

Dans ce contexte, l'objectif de cet article est de montrer qu'il est pertinent, justifié et intéressant de mobiliser le concept d'attachement pour qualifier la relation affective entre un consommateur et un lieu de service, au-delà des variables classiques utilisées par le marketing pour décrire les états affectifs. Une synthèse de la littérature sur l'attachement au lieu est d'abord proposée : elle met l'accent sur la méconnaissance de ses mécanismes. Des voies de recherche dans le cadre de la gestion d'un lieu de service sont ensuite proposées. Après avoir montré l'importance des recherches actuelles sur l'affect d'une part et sur les relations entre un consommateur et son environnement commercial d'autre part, cette partie souligne l'intérêt de mobiliser le concept d'attachement au lieu, notamment dans le cadre de la gestion d'un lieu de loisir. Ainsi, le recours au concept d'attachement au lieu serait particulièrement approprié aussi bien dans le cas des lieux de sortie (restaurants, bars) que dans celui des lieux culturels (cinémas, salles de spectacles), des lieux de vente « plaisir » (librairies, boutiques de mode) ou des sites touristiques. Enfin, une réflexion plus générale sur l'attachement au 
lieu dans la sphère du marketing témoigne de la richesse académique et managériale potentielle de ce terrain d'investigation.

\section{L'attachement au lieu: une revue de littérature pluridisciplinaire}

L'attachement au lieu n’a jamais été investigué dans le cadre des lieux de services, l'immense majorité des recherches portant sur l'étude du voisinage, de la maison, de la communauté ou des lieux de loisirs de plein air (parcs nationaux, forêts, rivières etc.). Faisant l'objet d'études dans plusieurs disciplines, à travers de nombreux points de vue et méthodologies, l'attachement au lieu concerne des lieux qui différent en termes de taille, d'expérience ou de tangibilité (Low et Altman, 1992), ce qui explique l’absence de définition générique du concept.

\subsection{De l’absence de définition unique à la précision du concept}

L'attachement au lieu ne dispose d'un véritable consensus ni du point de vue de sa définition, ni dans son approche méthodologique, ni même de son nom (Hidalgo et Hernandez, 2001). Cependant, si les définitions proposées aujourd'hui sont encore imprécises (Giuliani et Feldman, 1993) et ne permettent pas toujours facilement de différencier le concept d'attachement au lieu de concepts connexes proches, l'attachement au lieu comporte néanmoins un certain nombre de caractéristiques communes et singulières au concept (Schultz-Kleine et MenzelBaker, 2004). Elles permettent de proposer une définition valable quel que soit le type de lieu considéré. Ainsi, l'attachement au lieu est un lien affectif positif entre un individu et un lieu spécifique, ce dernier constituant pour l'individu une extension du soi. Se forgeant au travers d'interactions répétées au cours du temps entre l'individu et le lieu, l'attachement donne au lieu une valeur particulière, distincte de sa valeur utilitaire. Sa disparition est alors susceptible d'entraîner tristesse et manque. L'attachement au lieu varie en intensité et a pour nature de durer.

Si la littérature caractérise l'attachement au lieu par un certain nombre de propriétés, bien des questions sur le construit restent en suspens (Debenedetti, 2004), notamment celui de sa dimensionnalité. S’agit-il d'un concept intégré ou d'un concept dimensionnel ? Là encore, le débat est loin d'être clôt et cette focalisation sur les questions relatives à la définition du construit n’ont pas favorisé la production de connaissances nouvelles.

\subsection{Des mécanismes encore peu explorés}

Quels mécanismes régissent l'attachement au lieu ? Peu de recherches se sont intéressées aux antécédents, conséquences et facteurs d'influence du construit et l'analyse de la littérature montre que les résultats obtenus sont encore modestes.

D’abord, pourquoi s’attache-t-on à un lieu ? Les pistes données à ce jour sont peu consensuelles. La littérature sur l'attachement au lieu de résidence met en avant l'existence d'un préalable temporel à l'attachement (Hay, 1998 ; Hummon, 1992), même si la causalité de la relation n’est pas testée ni même clairement évoquée. L’âge, la position dans le cycle de vie ou la propriété du lieu y sont parfois 
ajoutées comme autres variables explicatives (Mesch et Manor, 1998). Dans le même ordre d'idée, Brown et Perkins (1992) proposent qu'une certaine stabilité constitue un terrain propice à l'instauration d'un attachement. Sans être clairement identifiés comme antécédents, le rythme de fréquentation, les expériences vécues sur le lieu (Moore et Graefe, 1994), le nombre d'occurrence de visites (Rubinstein et Parmelee, 1992) ou le besoin d'un contexte personnel, physique ou social, proche de l’idée d’expérience (Mesch et Manor, 1998), sont également évoqués.

S’attacher à un lieu implique-t-il des conséquences particulières pour l'individu ? Là encore, la littérature n'est guère explicite. En dehors des conséquences en terme d'attachement au lieu de résidence, qui se résument à une résistance au changement (Mesch et Manor, 1998), la littérature expose succinctement les thèmes également mis en avant dans les travaux sur l'attachement aux possessions, à savoir l'implication et l'engagement envers le lieu (Wakefield et al., 2001), la difficulté de substitution (Williams et al., 1992), l'apparition d'un sentiment de sécurité, l'établissement de routines et de rites ou la perturbation en cas de disparition du lieu d'attachement (Brown et Perkins, 1992).

Quelles variables sont susceptibles d'influencer la relation d'attachement entre un individu et un lieu ? D’abord, les femmes développeraient plus d'attachement au lieu que les hommes, quel que soit le type de lieu (Hidalgo et Hernandez, 2001). Ensuite, l'attachement au lieu augmenterait avec l'âge et / ou le temps (Hidalgo et Hernandez, 2001). Pour certains, l'attachement au lieu augmenterait également avec la variété des expériences vécues sur le lieu considéré (Jorgensen et Stedman, 2001) ou avec l'incapacité de l'individu à faire face à l'environnement (Riley, 1992). Enfin, il existe très peu de travaux faisant référence à l'influence de variables psychologiques sur l'attachement au lieu. Sans offrir plus de précision, Rubinstein et Parmelee (1992) évoquent l'influence de la personnalité et Riley (1992), pose la question de l'influence de l'humeur, faisant également émerger les influences possibles de la personnalité de l’individu.

Enfin, des échelles ont été développées pour mesurer l'attachement au lieu, principalement en sciences des loisirs. Cependant il n'existe pas d'échelle standardisée (Williams et al., 1992). De plus, les échelles disponibles partent souvent d'une conceptualisation floue de l'attachement au lieu et souffrent de nombreuses limites méthodologiques.

Force est donc de constater que la littérature ne permet pas d'appréhender le concept dans toute sa complexité, les connaissances restant limitées concernant les antécédents, les conséquences, les facteurs d’influence et la mesure du construit. Ainsi, l'attachement au lieu suggère de nombreux prolongements et de nombreuses pistes de réflexion, en particulier dans le contexte de la consommation, vierge de tout travaux. Ce concept constitue donc aujourd'hui une source importante de défis théoriques et de réflexions managériales dans le cadre de la gestion de la relation entre un consommateur et un lieu de service.

\section{L'attachement au lieu : un champ riche en voies de recherches}

Si l'attachement au lieu n'est pas à l'origine de recherches dans le cadre de la consommation, la littérature s'est en revanche intéressée d'une part aux liens 
affectifs dans leur diversité, d'autre part aux relations entre le consommateur et le lieu de service.

\subsection{L'affect et la relation consommateur / lieu de vente en marketing : des voies déjà en partie explorées}

L'affect fait en effet l'objet de recherches en marketing depuis qu'il est admis conceptuellement et empiriquement que les affects et les cognitions peuvent exister de manière indépendante (Zajonc et Markus, 1982). Filser (1996) rappelle que le marketing nécessite des outils conceptuels et méthodologiques pour analyser les décisions et les comportements dans le domaine du tourisme par exemple, où les modèles de comportement retenant les dimensions cognitives de la prise de décision trouvent leur limite. Aujourd'hui encore, peu de recherches en marketing mettent l'accent sur la nature des relations affectives qui s'instaurent entre le consommateur et le lieu. Cependant, envisager les états affectifs dans leur diversité (par exemple, en termes d'intensité ou de durée) semble important, puisque chaque affect implique des mécanismes spécifiques et des réponses comportementales différentes de la part du visiteur. Il est donc nécessaire d'examiner les états affectifs en se focalisant sur un affect particulier (Derbaix et Pham, 1989). Par ailleurs, la littérature montre que les termes d'affect, d'émotion, d'humeur ou d'attitude ont souvent été utilisés de manière inappropriées dans la littérature (Bagozzi, Gopinath et Nyer, 1999), notamment en raison de la diversité des définitions des concepts et des états qu'ils décrivent.

La littérature relative aux relations entre les lieux et les consommateurs concerne d'abord la fréquentation des points de vente. Les travaux montrent que les motifs liés à l'activité de shopping sont variés. Outre des motifs rationnels (recherche d'information, achat), qui constituent une part importante de la littérature, le consommateur fréquente les lieux de service pour des mobiles personnels (distraction, activité physique), sociaux (recherche de lien social, désir d'assimilation à un groupe) ou intrinsèques c'est-à-dire relatifs au plaisir de réaliser l'expérience de shopping pour elle-même (Bloch, Ridgway et Dawson, 1994 ; Holbrook et Hirschman, 1982). Nombre de ces motivations font donc référence à des dimensions affectives de la consommation, alors que les modèles de choix de magasin mettent en avant l'aspect cognitif de ce choix (Filser, 1994). Ensuite, les variables d'atmosphère (musique, odeurs, foule) montrent qu'il est possible d'influencer la relation affective au lieu de vente au-delà de l'impact des variables commerciales sur les réactions cognitives du consommateur (Cliquet, Fady et Basset, 2002). Enfin, l'état affectif peut également constituer un facteur de satisfaction du consommateur à l'égard de l'enseigne (Plichon, 1998 ; Lichtlé, Llosa et Plichon, 2001).

Les travaux déjà réalisés permettent de mieux comprendre l'existence d'un lien affectif entre un consommateur et un lieu de service mais ne font pas en revanche la distinction entre les différentes formes que ce lien peut revêtir. Envisager la relation affective entre le consommateur et son environnement commercial en mobilisant le concept d'attachement au lieu permet de combler ce manque en décrivant de manière fine un type spécifique d’affect. 


\subsection{Vers un cadre conceptuel ancré dans le contexte de la consommation et des lieux de loisirs.}

Si la littérature permet de proposer une définition générique claire de l'attachement au lieu, l'absence de recherches sur le concept dans le cadre de la consommation justifie la mise en place d'une méthodologie qualitative exploratoire permettant de décrire de manière fine l'attachement du consommateur au lieu de service.

Il s'agit d'aller au-delà de l'importance du temps et de l'occurrence de visite dans la création du lien d'attachement, de faire émerger des données qualitatives les réponses comportementales à l'attachement au lieu en dehors de celles qui sont proposées par la littérature et qui montrent leurs limites. Les conséquences pour l'individu doivent être précisées, détaillées et ancrées dans le contexte de la visite d'un lieu de service. Enfin, il s'agit de faire apparaître, au-delà des variables comme l'âge et le sexe, quelles autres variables individuelles ou quelles variables marketing (comme la qualité de service) sont susceptibles d'influencer la relation d'attachement entre un consommateur et un lieu de service. Par exemple, on peut imaginer que la confiance en soi peut influencer l'attachement au lieu. En effet, un individu avec une faible confiance en soi peut rechercher l'appui d'un lieu qui correspond à son soi idéal et bénéficie d'une reconnaissance ou d'un prestige qu'il ne pense pas posséder. Comment alors rassurer ce consommateur dans le cadre d'un lieu de service, lui donner un sentiment de sécurité ? Par le personnel, en instaurant un lien social perçu comme authentique ? Par l'aspect esthétique du lieu, en proposant un aménagement original et unique aux yeux du consommateur? On peut ensuite imaginer que la conséquence en sera une plus grande fidélité ou d'autres comportements de fréquentation spécifiques à l'attachement au lieu. Comme le soulignent Hidalgo et Hernandez (2001), il est nécessaire d'aller au-delà de la facette sociale de l'attachement au lieu. Quid alors de la dimension expérientielle de la visite : ne joue-t-elle pas un rôle important dans l'intégration du lieu dans le concept de soi du consommateur?

La question du terrain d'application de l'attachement au lieu est primordiale. Si la littérature pose que l'attachement concerne n'importe quel type de lieu, les exemples de lieux de service sont rares, sauf à considérer les lieux de récréation naturels étudiés en sciences des loisirs. Il est donc nécessaire de savoir si les lieux de service sont susceptibles de provoquer un attachement du consommateur et si oui, envers quels types de lieux de service l'attachement se développe prioritairement? Les premiers résultats d'une étude exploratoire utilisant plusieurs méthodologies et réalisée par l'auteur montrent que certains lieux de service sont plébiscités Il s'agit d'abord de lieux de loisirs, en particulier de lieux de sortie (cafés, restaurants, bars), de lieux culturels (cinémas, musées, salles de spectacles) et de lieux de vente " plaisir » (librairies, grands magasins, boutiques de mode, disquaires) ; ensuite de lieux touristiques. Ils se distinguent tous par une fréquentation exempte de contrainte.

\subsection{L'enjeu pour le marketing relationnel et le marketing du lieu de service}

En dehors du prolongement logique de cette revue de la littérature par une exploration du construit, cet article suggère de nombreuses voies de recherche 
relatives à la manière d'envisager l'attachement dans le cadre du marketing relationnel et du marketing des lieux de service et de loisirs. Après avoir proposé un cadre conceptuel résumant les apports de l'étude qualitative, on pourra s'intéresser à mesurer l'attachement des consommateurs aux lieux de loisirs en développant une échelle de mesure. Il pourra ensuite s'agir de s'intéresser au processus de formation de l'attachement au lieu, en mettant l'accent sur les antécédents et la question de son instrumentalisation par l'entreprise, les questions les plus évidentes étant pour le manager et le chercheur : comment créer de l'attachement? Est-ce réellement possible de créer de l'attachement envers un lieu de loisirs? En aval du processus, les conséquences comportementales et cognitives sur l'individu et la gestion de ces conséquences par le lieu de service et de loisirs constituent une autre voie possible. On pourra également s'intéresser aux différences individuelles entre les personnes attachées aux lieux de loisirs et les autres consommateurs et voir si dans des situations de consommation identiques, on constate des réactions différentes, et comment on peut les gérer. Enfin, on pourra s'intéresser au parallèle entre la dimension de l'attachement et le type de lieu : est-ce que la manière de s'attacher dépend de la nature du lieu d'attachement?

D'un point de vue plus managérial, l'attachement au lieu pose la question de l'intégration de cette forme de relation affective dans la stratégie du lieu de loisirs : est-ce que l'attachement au lieu est une variable sur laquelle investir et si oui, à quelles conditions ? Le cas échéant, sur quelle(s) dimension(s) de l'attachement au lieu jouer? Ensuite, il est probable que l'aspect social revête une importance particulière dans les lieux de sortie et les magasins à fort caractère hédoniste. En tenir compte passe par répondre à des questions du type : comment disposer d'une force de vente pérenne ? Comment faire adopter les valeurs et l'identité du lieu par le personnel ? Par ailleurs, l'étude qualitative a fait émerger que certains individus s'attachaient à leur lieu de travail. On peut donc s'interroger sur l'influence de l'employé ou du manager sur l'attachement du consommateur au lieu de loisirs. Toujours en relation avec la composante sociale du lieu, le manager peut vouloir s'intéresser au rôle d'ambassadeur des clients attachés : comment aider les clients à diffuser leur sentiment, en particulier dans le cas où le lieu ne bénéficie pas d'un emplacement géographique privilégié et/ou ne dispose pas d'un budget de communication important ? Enfin, l'attachement au lieu pose au manager la question de la relation au lieu par rapport à la relation à l'enseigne. Etre attaché au cinéma MK2 Quai de Loire, à Paris, diffère du lien que le consommateur peut éprouver pour «l'enseigne » MK2 en général. Comment, dans ces conditions, développer une activité de loisirs sans affecter le sentiment que le consommateur éprouve envers un lieu particulier, étant donné que l'enseigne rend le lieu moins particulier ? Doit-on alors gérer séparément le lien à l'enseigne et la relation avec le lieu au niveau local ? Quel espace de liberté de gestion accorder alors à un lieu de loisirs spécifique par rapport à l'enseigne dont il constitue un élément? On peut même se demander si, dans certains cas, comme celui des salles de cinéma, personnaliser la salle jusqu’à ne pas avoir de référence à une enseigne commune ne serait pas davantage synonyme d'authenticité, de proximité et donc de lien ? Cette question est souvent posée dans le domaine de la géographie humaine : est-il nécessaire d'uniformiser les lieux au risque d'en faire des « labyrinthes de similarités sans fin » (Relph, 1976, p141), dépourvus de sens pour le consommateur donc incapables de susciter un lien véritable et volontaire ? 


\section{Conclusion}

L'attachement au lieu a fait l'objet de recherches dans de nombreuses disciplines, notamment en sciences des loisirs, où l'on recense 7 articles sur le concept dans les deux revues les plus importantes (Leisure Sciences et Journal of Leisure Research) depuis 2000, sans compter les articles concernant les construits connexes. Cet intérêt pour l'attachement au lieu en sciences des loisirs est aujourd'hui relayé par le marketing puisqu'il apparaît, à la lumière de la littérature et des données qualitatives collectées par l'auteur, que les lieux de loisirs dans leur grande diversité constituent un terrain particulièrement propice à l'étude des liens affectifs entre un consommateur et un lieu. Or, cette recherche est la première en marketing qui s'intéresse à un concept jusqu'ici négligé par ce champ. L'attachement au lieu peut donc contribuer à l'explication des relations affectives qui s'instaurent entre les consommateurs et les lieux de service sur de nombreux terrains, de la gestion d'une librairie à celle d'un restaurant, de celle d'un monument historique à celle d'une salle de concert. Le recours à ce concept suggère enfin de nouvelles perspectives managériales dans la gestion de la relation avec le client dans le lieu de service ou de loisirs. 


\section{Bibliographie}

R. Bagozzi, M. Gopinath et P. Nyer, “The role of emotions in marketing”, Journal of the Academy of Marketing Science, Vol.27, n², p.184-206, 1999.

R. Belk, “Attachement to possessions”, in Altman et Low, Place Attachment, Plenum Press, New York, p.37-61, 1992.

P. Bloch, N. Ridgway et S. Dawson, “The shopping mall as a consumer habitat”, Journal of Retailing, Vol. 70, n²1, p.23-42, 1994.

B. Brown et D. Perkins, "Disruption in place attachment", in Altman et Low, Place Attachement, Plenum Press, New York, p.279-302, 1992.

G. Cliquet, A. Fady et G. Basset, Management de la Distribution, Dunod, Paris, 2002.

A Debenedetti, “Attachement au lieu : état de l'art et perspectives de recherche dans le cadre des lieux de loisir", Actes des $9^{\text {èmes }}$ Journées de Recherche en Marketing de Bourgogne, Marc Filser Ed., Dijon, section 8, 2-19, 4-5 novembre 2004.

C. Derbaix et M. Pham, "Pour un développement des mesures de l'affectif en marketing : synthèse des pré requis”, Recherche et Applications en Marketing, Vol.4, n4, p.71-87, 1989.

M. Filser, Le comportement du consommateur, Collection Précis de Gestion, Dalloz, Paris, 1994.

M. Filser, “Vers une consommation plus affective?”, Revue Française de Gestion, Vol. 110, p.90-99, septembre-octobre 1996.

M.V. Giulani et R. Feldman, "Place attachement in a developmental and cultural context”, Journal of Environmental Psychology, Vol.13, p.267-274, 1993.

R. Hay, "Sense of place in developmental context", Journal of Environmental Psychology, Vol.18, p.5-29, 1998.

M.C. Hidalgo et B. Hernandez, "Place attachement : conceptual and empirical questions”, Journal of Environmental Psychology, Vol.21, p.273-281, 2001

M. Holbrook et E. Hirschman, "The experiential aspects of consumption : consumer fantasies, feeling, and fun”, Journal of Consumer Research, Vol.9, p.132-140, September 1982.

D. Hummon, "Community Attachement : local sentiment and sense of place”, in Altman and Low, Place Attachement, Plenum Press, New York, p.253-278, 1992. 
B. Jorgensen et R. Stedman, "Sense of place as an attitude : lakeshore owners attitudes toward their properties", Journal of Environmental Psychology, Vol.21, p.233-248, 2001.

M.C. Lichtlé, S. Llosa et V. Plichon, "La contribution des différents éléments d'une grande surface alimentaire à la satisfaction du client", Recherche et Applications en Marketing, Vol.17, n²4, p.23-34, 2001.

S. Low, "Symbolic ties that bind : place attachment to the plaza", in Altman and Low, Place Attachement, Plenum Press, New York, p.165-185, 1992.

S. Low et I. Altman, "Place attachment : a conceptual inquiry", in Altman and Low, Place Attachement, Plenum Press, New York, p.1-12, 1992.

G. Mesch et O. Manor, "Social ties, environmental perception, and local attachment”, Environment and Behavior, Vol.30, n4, p.504-519, july 1998.

R. Moore L. et A. Graefe, "Attachments to recreation settings : the case of railtrail users”, Leisure Sciences, Vol.16, p.17-31, 1994.

V. Plichon, “La nécessité d’intégrer les états affectifs à l'explication du processus de satisfaction du consommateur", Actes du XIIIème Congrès de l'Association Française du Marketing, ed. B. Saporta, Bordeaux, IAE, p.671-694, 1998.

E. Relph, Place and placelessness, Pion, London, 1976.

R. Riley, “Attachment to the ordinary landscape”, in Altman and Low, Place Attachement, Plenum Press, New York, p.13-35, 1992.

R. Rubinstein et P. Parmelee, "Attachment to place and the representation of the life course by the elderly”, in Altman and Low, Place Attachement, Plenum Press, New York, p.139-161, 1992.

S. Schultz-Kleine et S. Menzel-Baker, “An integrative review of material possession attachment”, Academy of Marketing Science Review, Vol.1, p.1-35, 2004.

S. Wakefield, S. Elliot, D. Cole et J. Eyles, "Environmental risk and (re)action : air quality, health, and civic involvement in an urban industrial neighbourhood”, Health \& Place, Vol. 7, p.163-177, 2001

D. Williams, M. Patterson, J. Roggenbuck et A. Watson, "Beyond the commodity metaphor : examining emotional and symbolic attachment to place”, Leisure Sciences, Vol.14, p.29-46, 1992.

R. Zajonc. et H. Markus, "Affection and cognitive factors in preferences”, Journal of Consumer Research, Vol.9, p.123-131, september 1982. 